\title{
Bacteriophage HK97 head assembly: dramatic rearrangements in the context of icosahedral symmetry
}

\author{
ROGER W. HENDRIX \\ Department of Biological Sciences and Pittsburgh Bacteriophage Institute, University of Pittsburgh, Pittsburgh, PA15260, USA
}

The HK97 capsid is a $T=7$ structure that assembles from pentamers and hexamers of the $41 \mathrm{kD}$ (384 aa) subunit. This produces a rather unstable structure called Prohead I (PH-I). Carrying out the assembly reaction in vitro has allowed us to demonstrate that both pentamers and hexamers are required for successful assembly. However, with a point mutant of the subunit (E219K), which is only mildly defective in virus production, we can demonstrate efficient assembly of hexamers alone, to produce "Whiffleballs"-360 subunit structures that are identical to the 420 subunit PH-I except that they are missing the 12 pentamers. Attempts to resolve this apparent conflict in the data suggest a mechanism of $\mathrm{PH}-$ I assembly in which a conformational change in hexamers induced by an early step of assembly constrains subsequent steps, to result in the assembly of only capsids of the correct size. This model is currently under test.

If the phage maturation protease is present during assembly, it gets incorporated into the structure and removes the N-terminal 102 aa from each capsid subunit. This produces a substantially more stable structure (Prohead II) but without large-scale morphological changes.

PH-II then undergoes a dramatic conformational rearrangement into the mature Head, in which subunits substantially change their interactions with neighbours, the internal volume of the shell increases by two-fold, and parts of subunits intertwine with their neighbours. Finally, each subunit makes covalent bonds to two of its neighbours. The geometry of the crosslinking produces topologically linked covalent protein circles known as chainmail. Crosslink formation is autocatalytic, and the catalytic site for each crosslink is formed by bringing together parts of three subunits during the conformational rearrangement of PH-II. The conformational rearrangement is completed with different kinetics at the 7 different quasi-equivalent positions, and we have been able to follow some aspects of this from the differential kinetics of crosslink formation.

[This work is the result of an ongoing collaboration among the laboratories of R. Hendrix, A. Steven, J. Johnson and J. Conway]

\section{References}

[1] Gan, L., Conway, J.F., Firek, B.A., Cheng, N., Hendrix, R.W., Steven, A.C., Johnson, J.E. and Duda, R.L., 2004, Control of crosslinking by quaternary structure changes during bacteriophage HK97 maturation. Mol. Cell, 14, 1-20.

[2] Hendrix, R.W. and Duda, R.L., 1998, Bacteriophage HK97 head assembly: A protein ballet. Adv. Virus Res., 50, 235-288.

[3] Li, Y., Conway, J.F., Cheng, N., Steven, A.C., Hendrix, R.L. and Duda, R.L., 2005, Control of virus assembly: HK97 "Whiffleball" mutant capsids without pentons. J. Mol. Biol., (in press). 


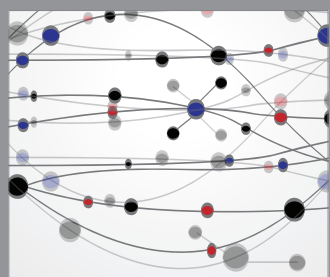

The Scientific World Journal
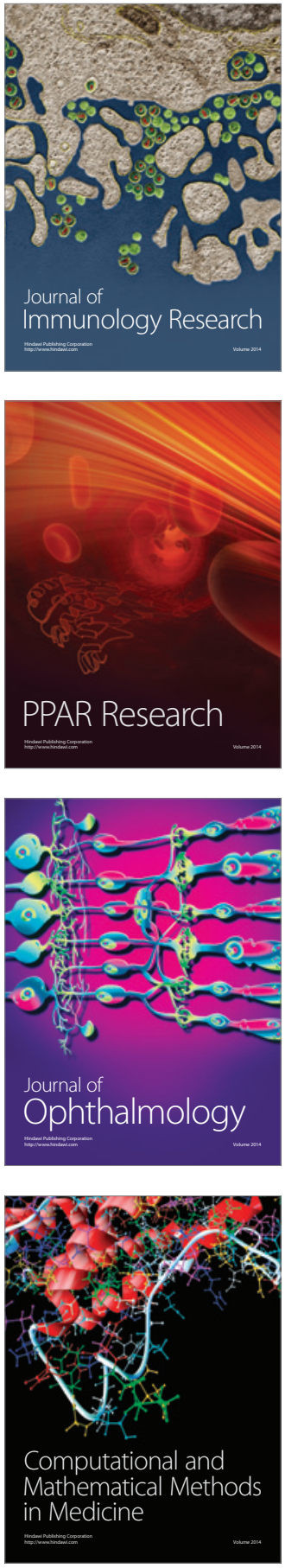

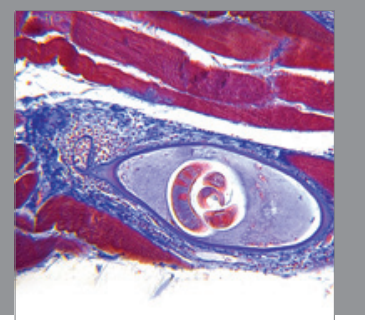

Gastroenterology

Research and Practice
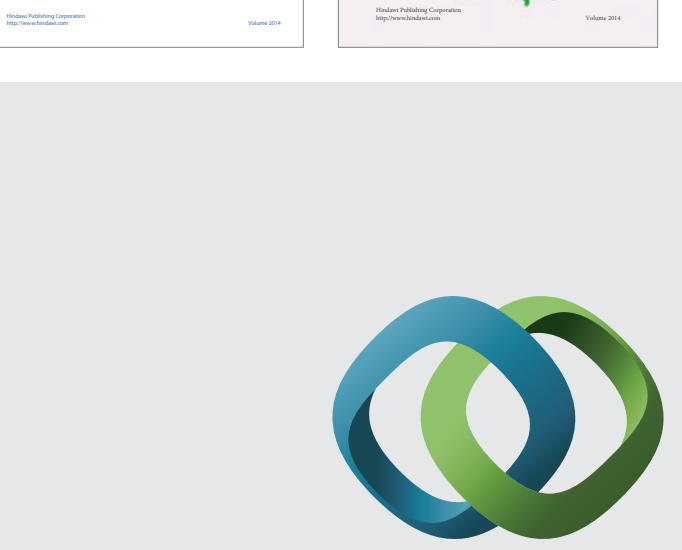

\section{Hindawi}

Submit your manuscripts at

http://www.hindawi.com
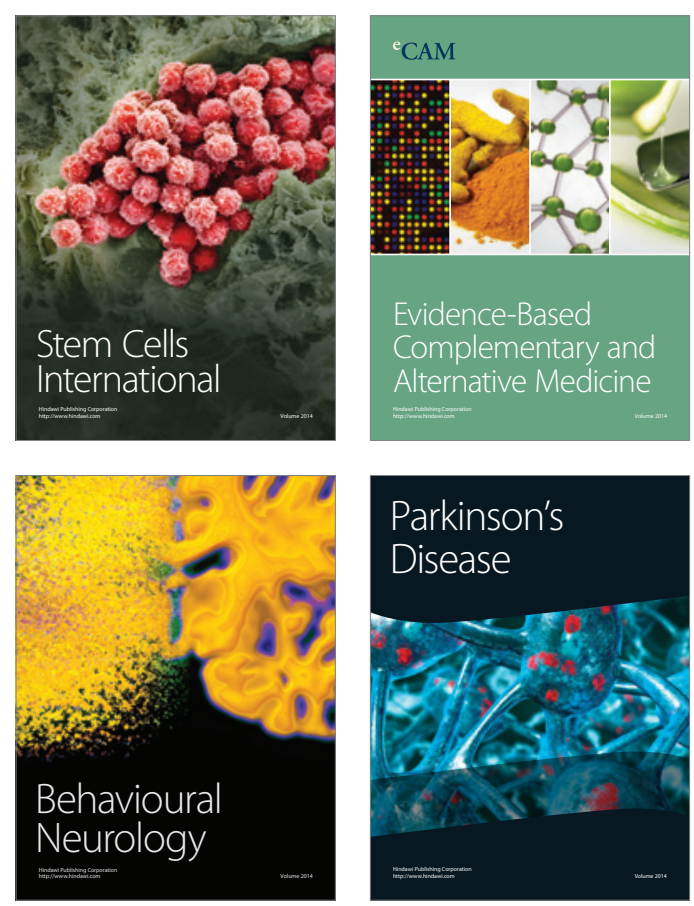

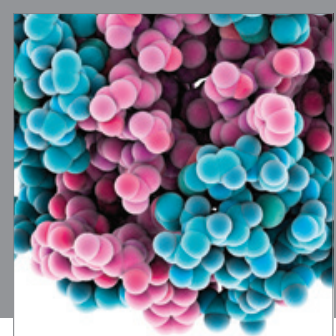

Journal of
Diabetes Research

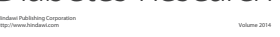

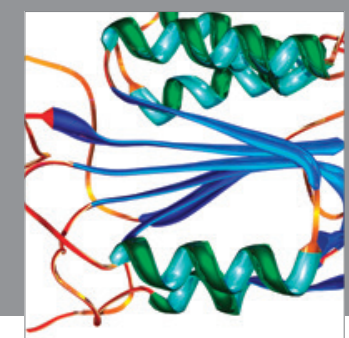

Disease Markers
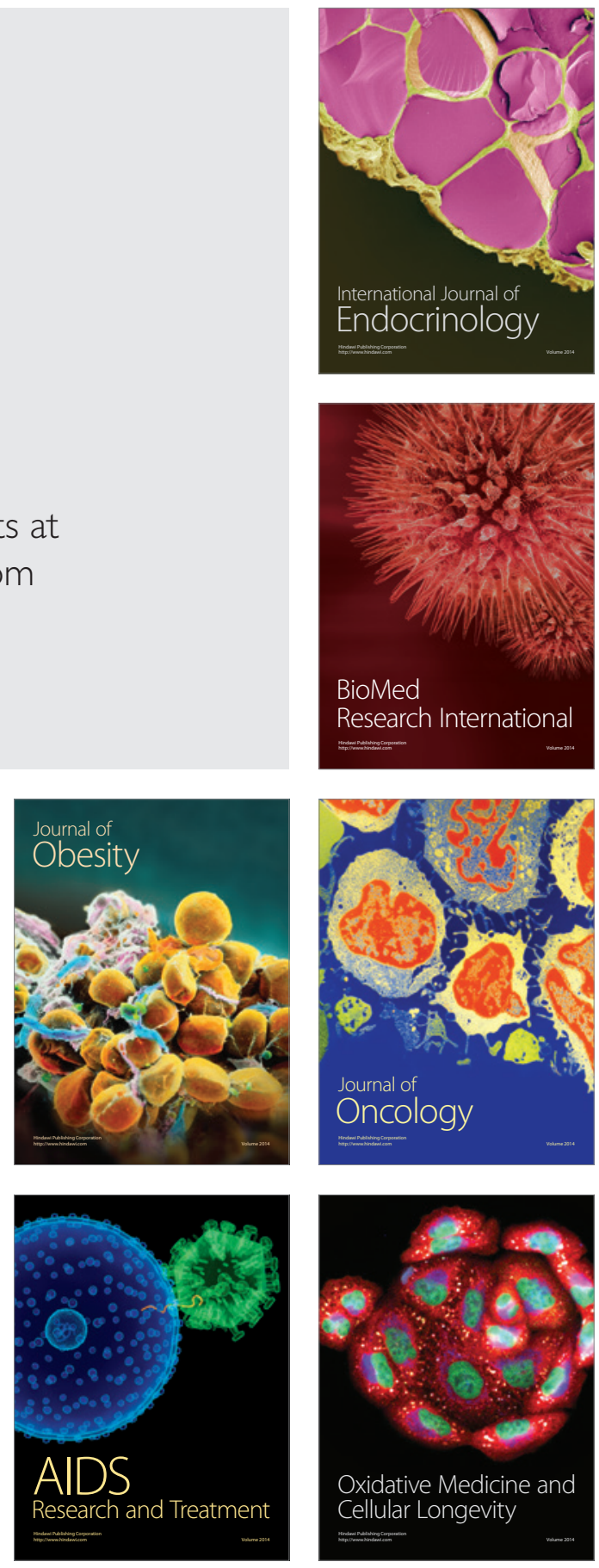